\title{
Repair versus shaving of partial-thickness articular-sided tears of the upper subscapularis tendon. A prospective randomized controlled trial
}

\author{
PIETRO RANDELLI ${ }^{1,2}$, PAOLO ARRIGONI ${ }^{2}$, ALBERTO ALIPRANDI ${ }^{2}$, SILVANA SDAO ${ }^{2}$, \\ VINCENZA RAGONE ${ }^{2}$, RICCARDO D'AMBROSI ${ }^{2}$, FILIPPO RANDELLI ${ }^{2}$, PAOLO CABITZA, ${ }^{1,2}$ \\ GIUSEPPE BANFI ${ }^{1,2}$
}

${ }^{1}$ Dipartimento di Scienze Biomediche per la Salute Università degli Studi di Milano, Italy

2 UO Ortopedia II, IRCCS Policlinico San Donato, San Donato Milanese, Milan, Italy

\begin{abstract}
Purpose: the purpose of this study was to evaluate whether treating partial-thickness articular-sided tears of the upper subscapularis (SSC) tendon with a dedicated suture anchor would result in an internal rotation strength improvement compared with simple shaving of the SSC tendon and footprint.

Methods: twenty-six patients with a limited SSC tendon tear (equal or inferior to the most superior centimeter) in association with a posterosuperior cuff lesion were prospectively randomized to two treatments: repair with a dedicated suture anchor versus shaving of the tendon and footprint. The patients also underwent long head of the biceps (LHB) treatment and posterosuperior cuff tear repair. In each patient the following parameters were measured both preoperatively and at a minimum follow-up of 2.5 years: strength in internal rotation in the bear-hug testing position (using a digital tensiometer), DASH score and Constant scores. MRI assessment of tendon healing was performed at the final follow-up.

Results: twenty of the 26 patients $(76 \%)$ were reviewed after a mean follow-up time of 42 months: 11 patients had undergone SSC tendon repair and nine simple shaving. At final follow-up no significant differences were found between the repaired and shaving
\end{abstract}

\footnotetext{
Corresponding Author:

Riccardo D'Ambrosi, MD

UO Ortopedia II, IRCCS Policlinico San Donato

Piazza Malan 1, San Donato Milanese, Italy

E-mail: riccardo.dambrosi@unimi.it
}

group in strength in internal rotation $(9.5 \pm 3.8 \mathrm{~kg}$ versus $10.3 \pm 5.4 \mathrm{~kg} ; \mathrm{p}=0.7)$. The DASH score and Constant score also failed to show significant differences between the two groups. Furthermore, no significant difference in SSC tendon healing rate was observed on MRI evaluation.

Conclusions: partial-thickness articular-sided tear of the upper SSC tendon in association with a posterosuperior rotator cuff repair and LHB treatment, when limited to the superior centimeter of the SSC tendon, shows a comparable performance in terms of strength in internal rotation either after simple shaving or a tendon-to-bone repair.

Level of evidence: Level II, prospective comparative study.

Keywords: subscapularis, shaving, suture anchor, arthroscopic cuff repair, MRI tendon healing.

\section{Introduction}

Subscapularis (SSC) tendon lesions can be traumatic or degenerative. Isolated SSC tendon lesions are relatively less frequent and mainly traumatic $(1,2)$. Partialthickness articular-sided tears of the SSC tendon are more common and often associated with posterosuperior cuff tears (3), and they are not well recognized by the standard available tests for SSC tendon tears (4). The optimal treatment for this specific tear pattern has not yet been defined.

Partial-thickness tears can progress, and increasing tear size associated with worsening pain underlies the need for the surgical repair (5). SSC tendon tear repair is 
advocated by several Authors who argued that anterior propagation of an SSC tendon tear is related to pseudoparalysis (6). Furthermore, SSC tendon tear repair may facilitate repair of the supraspinatus tendon (7).

Both symptomatic and asymptomatic partial-thickness tears have a decreased risk of tear progression compared with full-thickness tears (5). Furthermore, partialthickness SSC tendon tears, alone or in combination with supraspinatus tendon tears, were found to affect glenohumeral kinematics. However, partial-thickness SSC tendon tear repair does not restore kinematics to the intact level (8).

Several Authors have reported positive results with different approaches to these lesion patterns: shaving or use of a dedicated suture anchor repair. However, no consensus exists regarding the best treatment for lesions of this type (9-12).

The purpose of the present study was to evaluate whether dedicated suture anchor repair of partial-thickness articular-sided SSC tendon tears would determine an improvement in internal rotation strength compared with simple shaving of the SSC tendon and footprint. The study hypothesis was that maximum active strength would be higher in patients undergoing a suture anchor repair as opposed to simple shaving of the SSC tendon and footprint.

\section{Methods}

A prospective randomized controlled trial was conducted enrolling patients between December 2008 and June 2010.

All patients gave their informed consent prior to being included in the study. Patients were considered eligible for inclusion if they were scheduled for an arthroscopic supraspinatus or supraspinatus-infraspinatus repair and if the tear pattern was confirmed during diagnostic arthroscopy. Cases were enrolled if, on preoperative MRI, an associated partial-thickness articular-sided tear of the SSC tendon, defined as a lesion limited to the most superior centimeter of the tendon, was recognized. Arthroscopic examination was used to confirm the patient's eligibility for enrollment. As a further inclusion criterion, patients had to be able to reach an internal rotation equal or superior to the 3 rd lumbar vertebral body on preoperative physical examination.
Patients were excluded: a) if they were more than 75 years old; b) if they presented higher than grade 2 fatty degeneration of the most affected muscle between the supraspinatus and infraspinatus, according to the modified Goutallier classification (13); c) if a supraspinatus tear retraction of more than 1 centimeter, measured on the most central MRI coronal T2 view, was observed; d) if they were undergoing revision surgery, or e) if they presented associated systemic diseases such as rheumatoid arthritis or chronic inflammatory diseases.

Considering a difference of $3 \mathrm{~kg}$ in maximum active strength in internal rotation at the bear-hug testing position, the power analysis showed that at least 13 patients would be necessary in each group to detect this difference (4). These values were based on a power $(1-\beta)$ of 0.80 , a significance level of 0.05 (2sided), and a standard deviation of $2.5 \mathrm{~kg}$.

Twenty-six patients were included and randomized using a block randomized procedure. A block randomization list was generated by dedicated software (Stats Direct Ltd, Cheshire, UK). An independent operator, who was not involved in the surgical treatment, prepared sealed, opaque, numbered envelopes containing the treatment assignment. After a diagnostic arthroscopy to confirm the tear pattern patients were randomized into one of the two treatment groups.

Preoperatively all the patients were evaluated using the Constant score (14) and the national validated version of the DASH questionnaire (15). Maximum strength in internal rotation measured with a digital dynamometer (Kern, HBC series 20K50) was also recorded in the bear-hug testing position (4). Furthermore, each patient filled out an evaluation form including demographic characteristics. Rotator cuff tear pattern, including involvement of supraspinatus and infraspinatus tendons, was also recorded intraoperatively.

All the cases were treated arthroscopically by the same surgeon. Patients were positioned in a lateral decubitus position and treated according to a standard technique. The SSC tendon lesion was always addressed first. In all the cases a long head of the biceps (LHB) tenotomy or a tenodesis was performed, with a dedicated suture anchor positioned at the top of the bicipital groove. The supraspinatus/infraspinatus lesion was then addressed using a standard single-row suture anchor repair.

The treatment group underwent a direct SSC tendon 
repair with a double-loaded titanium suture anchor placed at the uncovered footprint level, with two tendon-to-bone sutures. The control group protocol consisted of shaving of the deep portion of the tendon and the tendon footprint.

Patients were discharged the day after surgery. The postoperative protocol was: four weeks wearing a sling followed by four weeks of passive stretching, and then active strengthening. During the first four weeks patients were allowed to remove the sling only for eating, dressing and bathing, and were recommended to avoid abduction, internal and external rotation.

The patients were routinely followed up in the early postoperative period. At a minimum of 2.5 years after surgery, patients were contacted for a final follow-up evaluation. They were evaluated by a single examiner, blind to their treatment, who recorded their Constant score, DASH score and strength in internal rotation in the bear-hug position.

An MRI examination was performed using a $1.5 \mathrm{~T}$ MRI unit (Magnetom Sonata Maestro Class; Siemens Medical Solution, Erlangen, Germany). The shoulder was placed in a flexible coil with the arm in the neutral position. The imaging protocol included a basal acquisition using Axial Turbo Spin-Echo (TSE), T1-weighted (T1W), coronal TSE T2-weighted (T2W), and oblique sagittal turbo inversion recovery magnitude (TIRM) sequences. The T1W and T2W sequences in axial and coronal planes were used in order to reduce metal artifacts resulting from the anchor placed on the lesser tubercle, and the TIRM sequences were used to identify and highlight spongy edema (Tab. 1).

Healing of the SSC tendon was classified considering the MRI axial and sagittal oblique planes of the most proximal available image, and $3.5 \mathrm{~mm}$ was taken as a normal thickness on the lesser tubercle (16). The coronal plane imaging served to detect any retraction of the re-torn tendon. Patients were asked to undergo
MRI examination at our institution. Images were analyzed by two radiologists with, respectively, 10 and 4 years' experience in musculoskeletal radiology. In patients unable to undergo the examination at our institution, MRI examinations performed elsewhere were acceptable. In such cases SSC tendon healing was graded afterwards. SSC tendons were classified, according to their appearance, as (17): Grade 1=tendon erosion; erosion of SSC tendon fibers (equal to or less than $30 \%$ of $3.5 \mathrm{~mm}$ ), without tendon retraction (Fig. 1); Grade 2 = partial tear; partial-thickness tear (more than $30 \%$ ) of the upper fibers of the SSC tendon (Figs. 2, 3); Grade 3= complete tear; detachment of the SSC tendon fibers from the lesser tubercle.

Statistical analysis was carried out using SPSS version 17 (SPSS Inc., Chicago, IL, USA). Differences between the treatment and control group for continuous variables were evaluated with the unpaired Student ttest or Mann-Whitney test, according to the characteristics of the data distribution. Differences for categorical variables were analyzed with a chi-square test or Fisher's exact test. The inter-observer agreement between the two radiologists was calculated by Cohen's kappa statistics. For all analyses, a p-value $<0.05$ was considered statistically significant.

\section{Results}

Of the 26 patients enrolled and randomized, $6(2$ from the treatment group and 4 from the control group) were not available for final follow-up. The study group thus comprised 20 patients (drop-out rate: $23 \%$ ).

Eleven patients from the repaired group and 9 from the shaving group completed the clinical and radiological follow-up. The two groups were comparable in terms of age at surgery, shoulder dominance, average

Table 1. Technical parameters of magnetic resonance examination.

\begin{tabular}{|c|c|c|c|c|c|}
\hline Sequences & $* \mathrm{TR}$ & $* \mathrm{TE}$ & $* \mathbf{I R}$ & SLICE THICKNESS (mm) & MATRIX \\
\hline Axial TSE T1w & 393 & & & 3 & $320 \times 320$ \\
\hline Coronal TSE T2w & 3000 & 76 & & 3 & $320 \times 320$ \\
\hline Sagittal TIRM & 4200 & 21 & 130 & 3 & $320 \times 320$ \\
\hline
\end{tabular}

$* \mathbf{T R}=$ repetition time $* \mathbf{T E}=$ echo time $* \mathbf{I R}=$ inversion recovery time 


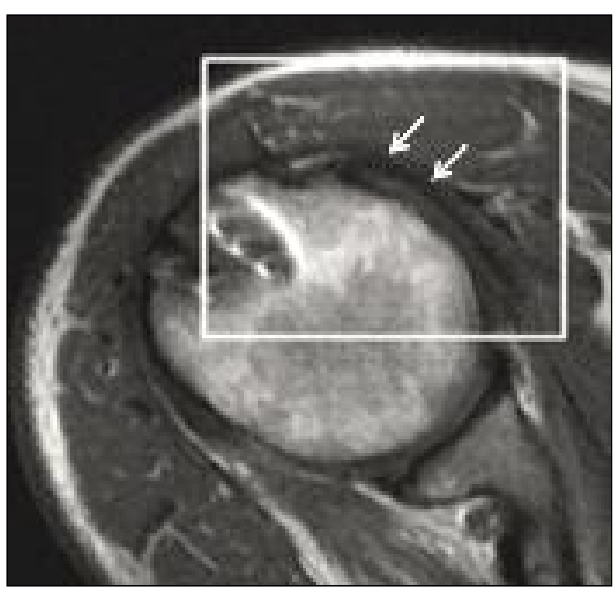

Figure 1. Axial T1 TSE image. Grade 1 lesion; white arrows show the SSC tendon at its insertion on lesser tubercle.

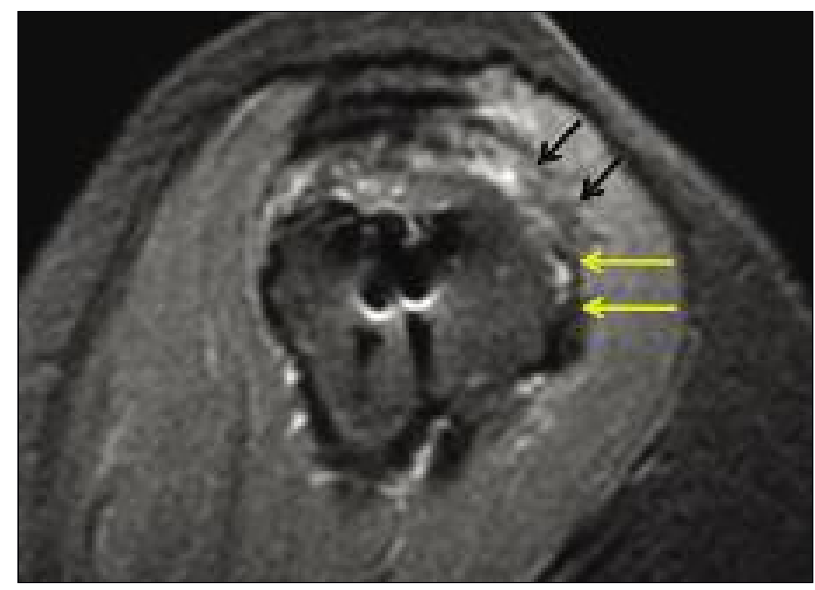

Figure 2. Sagittal TIRM image. Grade 2 lesion. Black arrows indicate the partial tear. Yellow arrows indicate the residual inferior fibers of the subscapularis tendon.

time from surgery to follow-up, BMI, rotator cuff tear pattern, and incidence of smokers and of diabetics $(\mathrm{p}>0.05)$ (Tab. 2). The two groups were also comparable for preoperative Constant score $(43.7 \pm 24 v$ s $36.4 \pm 21)$, DASH score (37.8 \pm 26 vs $46.2 \pm 15)$, and strength in internal rotation $(4.3 \pm 4$ vs $2.8 \pm 4)$ $(\mathrm{p}>0.05)$. All the patients underwent LHB tenotomy apart from one member of the control group who underwent tenodesis. This difference was not significant $(\mathrm{p}>0.05)$.

At follow-up no significant differences were found between the repaired and shaving group in terms of strength in internal rotation $(9.5 \pm 3.8 \mathrm{~kg}$ vs $10.3 \pm 5.4$ $\mathrm{kg} ; \mathrm{p}=0.7)$. Similarly, no significant differences bet-

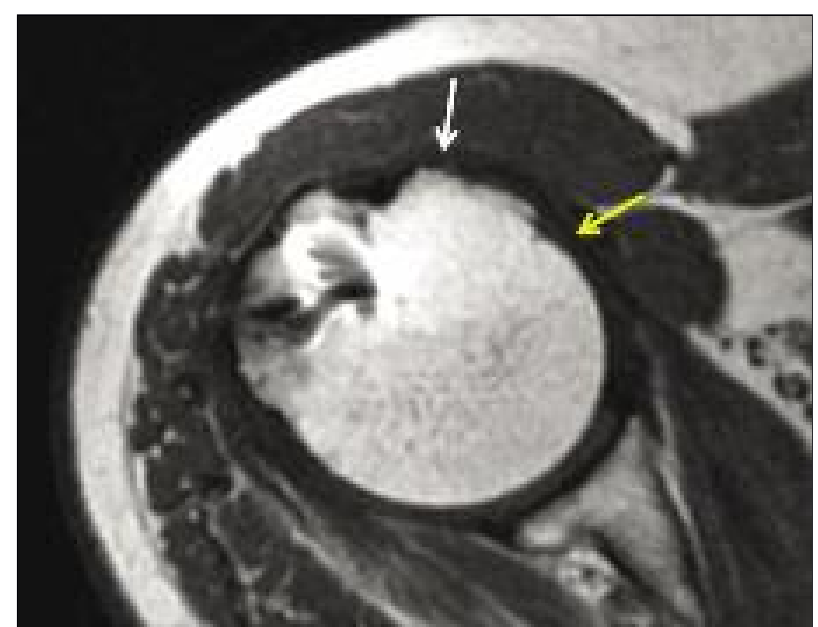

Figure 3. Axial TSE T1W image. Grade 2 lesion; white arrow shows partial interruption of subscapularis tendon fibers. Yellow arrow shows the residual fibers medially to the lesser tubercle.

ween the two groups were found in the DASH or Constant scores (Tab. 3).

The two radiologists showed good agreement with regard to the MRI assessment ( $\mathrm{k}$ coefficient $=0.767)$. Three patients performed the MRI examination externally and were graded as 1 .

Eleven out of 20 patients were assigned to grade 1 and nine to grade 2 . No patient presented a complete detachment of the SSC tendon fibers from the lesser tubercle (grade 3).

Distribution of SSC tendon healing rate was not significantly different between the two groups at final follow-up ( $\mathrm{p}=0.4)$ (Tab. 4).

\section{Discussion}

The main purpose of the present study was to determine whether use of a dedicated suture anchor to repair partial-thickness articular-sided tears of the upper SSC would result in an improvement internal rotation strength compared with simple shaving of the SSC tendon and footprint. No significant difference in internal rotation strength was observed between the groups. Therefore, the null hypothesis of the study was not rejected. Furthermore, no betweengroup differences were found in the Constant score, DASH score, or tendon healing rate on MRI. Lesions of the SSC tendon are disabling due to pain and to 
Table 2. Baseline and demographic characteristics.

\begin{tabular}{llll}
\hline & Repaired group (N.=11) & Shaving group (N.=9) & p-value \\
\hline Follow-up (months) & $41.7 \pm 6$ & $43 \pm 6$ & 0.4 \\
Age & $65 \pm 6$ & $65 \pm 6$ & 0.9 \\
BMI & $29 \pm 6$ & $28 \pm 4$ & 0.9 \\
Sex - N. Male (\%) & $5(45.4)$ & $4(44.4)$ & 1 \\
N. dominant shoulders (\%) & $7(63.6)$ & $7(77.7)$ & 0.6 \\
N. non-smokers (\%) & $10(91)$ & $9(100)$ & 1 \\
N. not diabetic (\%) & $11(100)$ & $9(100)$ & n.a. \\
N. SSP tendon tear & $11(100)$ & $9(100)$ & n.a. \\
N. ISP tear & $6(54.5)$ & $4(44.4)$ & 0.6 \\
Internal rotation strength (kg) & $4.3 \pm 4$ & $2.8 \pm 4$ & 0.4 \\
Constant score, pre-op. & $43.7 \pm 24$ & $36.4 \pm 21$ & 0.5 \\
DASH score pre-op & $37.8 \pm 26$ & $46.2 \pm 15$ & 0.5 \\
\hline
\end{tabular}

Values are expressed as mean \pm standard deviation or number of cases (percentage); n.a., not applicable.

Table 3. Clinical outcomes in the treatment and control group before surgery and at a minimum of 2.5 years of follow-up.

\begin{tabular}{llll}
\hline & Repaired group (N.=11) & Shaving group (N.=9) & p-value \\
\hline Internal rotation strength (kg) & $9.5 \pm 3.8$ & $10.3 \pm 5.4$ & 0.7 \\
Constant score & $81 \pm 15$ & $88.8 \pm 13.8$ & 0.2 \\
Subjective Constant score & $27.8 \pm 6.7$ & $30.8 \pm 5.8$ & 0.3 \\
Objective Constant score & $53.2 \pm 10.5$ & $58 \pm 8.3$ & 0.3 \\
DASH score & $17.2 \pm 15$ & $7.3 \pm 12$ & 0.1 \\
\hline
\end{tabular}

Table 4. Distribution of grades of subscapularis tendon tears.

\begin{tabular}{llll}
\hline & $\begin{array}{l}\text { Repaired group } \\
\text { (N.=11) }\end{array}$ & $\begin{array}{l}\text { Shaving group } \\
(\mathbf{N . = 9 )}\end{array}$ & p-value \\
\hline Grade 1 & $5(45.5 \%)$ & $6(66.7 \%)$ & 0.4 \\
Grade 2 & $6(54.5 \%)$ & $3(33.3 \%)$ & \\
Grade 3 & $0(0 \%)$ & $0(0 \%)$ & \\
\hline
\end{tabular}

biomechanical imbalance resulting in loss of active motion $(2,18,19)$. Arthroscopy can be used to evaluate both the intra- and extra-articular surfaces, and it allows an accurate diagnosis as it is able to define and distinguish between lesions. Combined tears of the SSC, supraspinatus and infraspinatus tendons are frequently associated with proximal humeral migration and loss of the overhead function. Arthroscopic repair of these massive tears can produce durable restoration of overhead function (18). Our study evaluated 20 patients affected by a limited lesion of the superior centimeter of the SSC tendon together with a posterosuperior tear. Our data show that not all SSC tendon lesion patterns need a specific suture anchor repair. Postoperative use of a sling in a resting posi- tion together with tendon debridement can result in a comparable healing rate.

Previous case series have shown improvement of clinical outcomes after arthroscopic repair of SCC tendon tears showing different lesion patterns. A retrospective review of arthroscopic SSC tendon repairs with minimum follow-up of seven years (20) reported good or excellent results in $83.3 \%$ of cases with $92.4 \%$ of patients returning to normal activities. This study showed that improvement of functional outcome after arthroscopic repair of SSC tendon tears is maintained in the long term. Lanz et al. (11), at 2-to-4-years of follow-up, concentrated on large and complete SSC tendon lesions and reached similar conclusions. Musil et al. (21) evaluated 23 patients, with a mean age of 55 years (range, 37-74), after arthroscopic repair of the SSC tendon. At an average follow-up of 24 months, Constant and UCLA scores had improved from 44.4 to 84.75 , and from 13.8 to 28.6, respectively. All the patients reported improvement and expressed their willingness to undergo the same surgery on the other shoulder, if need be. A recent study (22) evaluated pre- and postoperative clinical and structural outcome data on arthro- 
scopically treated anterosuperior rotator cuff tears involving the supraspinatus and SSC tendons. The mean follow-up was 16.9 months (range, 13-24 months). Compared with preoperative values, significant postoperative improvements were recorded in Constant score, forward flexion, strength and pain $(p<0.01)$. The patients also showed significant improvements in both the lift-off and belly press tests $(\mathrm{p}<0.001)$. Mean postoperative patient satisfaction was 7.9 (range, 5-10) with $10(59 \%)$ out of 17 patients being extremely satisfied. Two of the 17 patients with an anterosuperior rotator cuff tear had confirmed re-tears of the supraspinatus tendon (1 partial and 1 full-thickness) with no re-tear of any of the SSC tendon repairs. Our study is the first to compare direct suturing versus simple shaving in patients presenting a specific pattern of SSC tendon damage. Some Authors have highlighted good long-term follow-up results in patients with SSC tendon tears, but no comparison of these two different types of treatment is available. Direct repair can certainly benefit patients, especially those with massive lesions, but we do not have long-term results on lesions limited to the upper profile, and in particular, on lesions treated with simple tendon shaving.

Kim et al. (23) evaluated a total of 29 shoulders with an isolated partial articular-surface tear of the SSC tendon treated with arthroscopic intra-articular repair using suture anchors. LHB tenodesis was performed in 16 patients. They concluded that arthroscopic intraarticular repair of a partial-thickness articular-sided tear of the SSC tendon is an effective procedure that spares the intact tendon attachment in the bursal surface. Edwards et al. (12) instead evaluated the treatment of properly selected patients with isolated SSC tears by arthroscopic debridement and release of the LHB. They found that the combination of arthroscopic debridement and LHB tenotomy in the treatment of SSC tears with LHB disease in selected patients yielded good objective improvement and a high degree of patient satisfaction.

All the patients in our study underwent assessment of strength in internal rotation measured with a digital dynamometer in the bear-hug testing position. The bear-hug examination performed at specific degrees of shoulder flexion has been shown to be valuable for assessing SSC muscle function (24), as it is the most sensitive of all the SSC tests (82\%) (25). At $45^{\circ}$ of shoulder flexion, this test has proven to be specific for the upper SSC tendon $(4,24)$. This evidence was not confirmed by a recent electromyographic study (26), performed to ascertain ideal shoulder positions for isolating the SSC muscle while performing the bearhug, belly-press and lift-off tests. The level of SSC muscle activation was similar between all the tests. On the basis of these results, there is no ideal position for selectively testing the tendon portions. Nevertheless, dynamometric strength in internal rotation, the parameter measured in our methods, remains the most objective indicator of muscle-tendon function.

All the patients in our study underwent postoperative MRI. Pfirrmann et al. (27) first assesses the use of MR arthrography in the detection and grading of SSC tendon tears and showed the technique to be accurate for this purpose. In a recent systematic approach, a consistent finding was that the larger SSC tendon tears are the more easily they are recognized on MRI scans (28). This evidence supports the possibility of casual detection of partial-thickness articular-sided lesions during arthroscopy. Another recent study examined the accuracy of MRI in SSC tendon evaluation. A total of 244 patients were included with a total of 25 SSC tendon tears subclassified as partial-thickness $(\mathrm{n}=16)$ and full-thickness $(\mathrm{n}=9)$. A significant association was found between the presence of a partial SSC tendon tear during arthroscopy and a fluid-like signal within the tendon in more than one imaging plane $(p<0.001)$, with an accuracy of $90 \%$. The Authors concluded that the radiologist's diagnostic accuracy is improved by an understanding of certain imaging pitfalls and the presence of fluid-like signals in multiple imaging planes (29). Our MRI results show that partial tears of the SSC tendon treated by simple shaving do not progress to complete lesions, even without a formal arthroscopic repair.

One major limitation of the present study is certainly the drop-out rate that reduced the level of evidence. The difference of $3 \mathrm{~kg}$ in internal rotation strength between the groups, used to calculate the power of the analysis, can be considered large. This value was adopted assuming that the behavior of the control cases (shaving) would correspond to the worst scenario of a complete re-tear of the SSC tendon. The data to support this value are based on previously available studies (4). The results of this study may be considered applicable only to limited (less than or equal to the superior centimeter) partial-thickness articular-sided SSC tendon tears. Our data do not support the appli- 
cation of this approach in cases with larger lesions. Other limitations are that we considered a single measurement of strength rather than an average result from a repeated series, and also our decision to use the $45^{\circ}$ flexion bear-hug position. This position has not been confirmed as the most accurate to evaluate the upper SSC tendon $(26,30,31)$. The MRI analysis was not blind to the radiologist by definition: the presence of suture anchors indicated the treatment arm of each patient. This represented another unavoidable bias. In conclusion, partial-thickness articular-sided tear of the upper SSC tendon in association with a posterosuperior rotator cuff repair and LHB treatment, when limited to the superior centimeter, shows comparable performance in terms of strength in internal rotation after either simple shaving or a tendon-to-bone repair.

\section{References}

1. Clark RJ, Marchessault J, Sizer PS Jr, et al. Isolated traumatic rupture of the subscapularis tendon. J Am Board Fam Pract. 2002;15:304-308.

2. Wright JM, Heavrin B, Hawkins RJ, et al. Arthroscopic visualization of the subscapularis tendon. Arthroscopy. 2001;17:677684.

3. Sakurai G, Ozaki J, Tomita Y, et al. Incomplete tears of the subscapularis tendon associated with tears of the supraspinatus tendon: cadaveric and clinical studies. J Shoulder Elbow Surg. 1998;7:510-515

4. Barth JR, Burkhart SS, De Beer JF. The bear-hug test: a new and sensitive test for diagnosing a subscapularis tear. Arthroscopy. 2006;22:1076-1084

5. Tashjian RZ. Epidemiology, natural history, and indications for treatment of rotator cuff tears. Clin Sports Med. 2012;31:589604.

6. Ladermann A, Collin P, Walch G. Correlation of the involved compartments of massive rotator cuff tear and loss of active shoulder range of motion. Knee Surg Sports Traumatol Arthrosc. 2012;20:S1-3.

7. Ticker JB, Burkhart SS. Arthroscopy. Why repair the subscapularis? A logical rationale. 2011;27:1123-1128.

8. Yoo JC, McGarry MH, Jun BJ, et al. The influence of partial subscapularis tendon tears combined with supraspinatus tendon tears. J Shoulder Elbow Surg. 2014;23:902-908.

9. Grueninger P, Nikolic N, Schneider J, et al. Arthroscopic repair of traumatic isolated subscapularis tendon lesions (Lafosse Type III or IV): a prospective magnetic resonance imaging-controlled case series with 1 year of follow-up. Arthroscopy. 2014;30:665-672.

10. Toussaint B, Audebert S, Barth J, et al. Arthroscopic repair of subscapularis tears: preliminary data from a prospective multicentre study. Orthop Traumatol Surg Res. 2012;98:S193-200.

11. Lanz U, Fullick R, Bongiorno V, et al. Arthroscopic repair of large subscapularis tendon tears: 2- to 4-year clinical and radiographic outcomes. Arthroscopy. 2013;29:1471-1478.

12. Edwards TB, Walch G, Nové-Josserand L, et al. Arthroscopic debridement in the treatment of patients with isolated tears of the subscapularis. Arthroscopy. 2006;22:941-946.

13. Fuchs B, Weishaupt D, Zanetti M, et al. Fatty degeneration of the muscles of the rotator cuff: assessment by computed tomography versus magnetic resonance imaging. J Shoulder Elbow Surg. 1999;8:599-605.

14. Constant CR, Murley AH. A clinical method of functional assessment of the shoulder. Clin Orthop Relat Res. 1987;214:160164.

15. Hudak PL, Amadio PC, Bombardier C. Development of an upper extremity outcome measure: the DASH (disabilities of the arm, shoulder and hand) [corrected]. The Upper Extremity Collaborative Group (UECG). Am J Ind Med. 1996;29:602-608.

16. D’Addesi LL, Anbari A, Reish MW, et al. The subscapularis footprint: an anatomic study of the subscapularis tendon insertion. Arthroscopy. 2006;22:937-940.

17. Itoi E, Hsu HC, Carmichael SW, et al. Morphology of the torn rotator cuff. J Anat. 1995;186:429-434.

18. Burkhart SS, Tehrany AM. Arthroscopic subscapularis tendon repair: technique and preliminary results. Arthroscopy. 2002;18:454-463.

19. Kuntz AF, Raphael I, Dougherty MP, et al. Arthroscopic subscapularis repair. J Am Acad Orthop Surg. 2014;22:80-89.

20. Denard PJ, Jiwani AZ, Lädermann A, et al. Long-term outcome of a consecutive series of subscapularis tendon tears repaired arthroscopically. Arthroscopy. 2012;28:1587-1591.

21. Musil D, Sadovský P, Stehlík J. Arthroscopic repair of subscapularis tendon tear. Acta Chir OrthopTraumatol Cech. 2010;77:228-234

22. Schnaser E, Toussaint B, Gillespie R, et al. Arthroscopic treatment of anterosuperior rotator cuff tears. Orthopedics. 2013;36:1394-1400.

23. Kim SH, Oh I, Park JS, et al. Intra-articular repair of an isolated partial articular-surface tear of the subscapularis tendon. Am J Sports Med. 2005;33:1825-1830.

24. Chao S, Thomas S, Yucha D, et al. An electromyographic assessment of the "bear hug": an examination for the evaluation of the subscapularis muscle. Arthroscopy. 2008;24:1265-1270.

25. Barth J, Audebert S, Toussaint B, et al. Diagnosis of subscapularis tendon tears: are available diagnostic tests pertinent for a positive diagnosis? Orthop Traumatol Surg Res. 2012;98:S178185.

26. Pennock AT, Pennington WW, Torry MRI, et al. The influence of arm and shoulder position on the bear-hug, belly-press, and lift-off tests: an electromyographic study. Am J Sports Med. 2011;39:2338-2346.

27. Pfirrmann CW, Zanetti M, Weishaupt D, et al. Subscapularis tendon tears: detection and grading at MR arthrography. Radiology. 1999;213:709-714.

28. Adams CR, Brady PC, Koo SS, et al. A systematic approach for diagnosing subscapularis tendon tears with preoperative magnetic resonance imaging scans. Arthroscopy. 2012;28:1592-1600.

29. Gyftopoulos S, O'Donnell J, Shah NP, et al. Correlation of MRI with arthroscopy for the evaluation of the subscapularis tendon: a musculoskeletal division's experience. Skeletal Radiol. 2013;42:1269-1275.

30. Lin L, Yan H, Xiao J, et al. Internal rotation resistance test at abduction and external rotation: a new clinical test for diagnosing subscapularis lesions. Knee Surg Sports Traumatol Arthrosc. 2015;23:1247-1252.

31. Yoon JP, Chung SW, Kim SH, et al. Diagnostic value of four clinical tests for the evaluation of subscapularis integrity. J Shoulder Elbow Surg. 2013;22:1186-1192. 